\title{
Conditions for up-down asymmetry in the core of tokamak equilibria
}

\author{
Paulo Rodrigues* and Nuno F. Loureiro \\ Associação Euratom-IST, Instituto de Plasmas e Fusão Nuclear, \\ Instituto Superior Técnico, Universidade de Lisboa, 1049-001 Lisboa, Portugal. \\ Justin Ball and Felix I. Parra \\ Plasma Science and Fusion Center, Massachusetts Institute of Technology, Cambridge, Massachusetts, USA.
}

(Dated: September 14, 2021)

\begin{abstract}
A local magnetic equilibrium solution is sought around the magnetic axis in order to identify the key parameters defining the magnetic-surface's up-down asymmetry in the core of tokamak plasmas. The asymmetry is found to be determined essentially by the ratio of the toroidal current density flowing on axis to the fraction of the external field's odd perturbation that manages to propagate from the plasma boundary into the core. The predictions are tested and illustrated first with an analytical Solovev equilibrium and then using experimentally relevant numerical equilibria. Hollow current-density distributions, and hence reverse magnetic shear, are seen to be crucial to bring into the core asymmetry values that are usually found only near the plasma edge.
\end{abstract}

PACS numbers: 52.25.Xz, 52.55.Fa, 52.65.Kj, 28.52.Av

Introduction.-Plasma turbulence is known to degrade particle and energy confinement in fusion devices, with serious consequences on their performance [1]. However, turbulent transport can be reduced, or even suppressed, in toroidally rotating plasmas by velocity gradients [16]. In the absence of external momentum sources, spontaneous (or intrinsic) plasma rotation may arise due to the momentum flux induced by symmetry breaking along magnetic field lines [7-11]. One such symmetry breaking mechanism is yielded by up-down asymmetric equilibria. Unfortunately, early assessments have found that the asymmetry due to the externally shaped plasma boundary largely fails to propagate in to the core [9-11]. The benefits of the induced momentum flux thus appear to be restricted to the outer part of the plasma, with limited success in reducing turbulent transport levels.

In this Letter, a local analytic equilibrium is developed near the magnetic axis in order to understand the factors on which the shape of the magnetic surfaces depends and how the asymmetry may be enhanced. We find that reverse magnetic shear configurations can significantly increase the asymmetry on axis and are therefore expected to extend the asymmetry-induced momentum flux deep into the core of tokamak plasmas, thereby improving its confinement properties.

Local on-axis equilibria.-The distribution of the poloidal-field flux $\psi$, normalized to an arbitrary constant $\psi_{\mathrm{N}}$, in the poloidal section of axisymmetric devices is given by the Grad-Shafranov (GS) equation $[12,13]$

$$
-\frac{1}{r} \frac{\partial}{\partial r} \frac{r}{R} \frac{\partial \psi}{\partial r}-\frac{1}{r^{2}} \frac{\partial}{\partial \theta} \frac{1}{R} \frac{\partial \psi}{\partial \theta}=R \dot{p}(\psi)+\frac{\dot{Y}(\psi)}{R}
$$

if centrifugal effects are neglected. The static pressure $p(\psi)$ and the squared poloidal current $Y(\psi)$ are normalized to $\mu_{0}^{-1} \psi_{\mathrm{N}}^{2} /\left(a R_{0}\right)^{2}$ and $8 \pi^{2} \psi_{\mathrm{N}}^{2} /\left(\mu_{0} a\right)^{2}$ respectively, the dots denote flux derivatives $d / d \psi$, and $(r, \theta, \phi)$ are right-handed coordinates with the origin displaced by $R_{0}$ from the tokamak's symmetry axis: The distance $r$ to the origin is normalized to $a, \theta$ is a poloidal angle measured from the midplane's high-field side, $\phi$ is the toroidal angle, and $\varepsilon=a / R_{0}$ is the inverse aspect ratio, with $a$ and $R_{0}$ the tokamak's minor and major radii. The distance $R$ to the symmetry axis and the height $Z$ above the midplane, both normalized to $R_{0}$, are

$$
R=1-\varepsilon r \cos \theta \text { and } Z=\varepsilon r \sin \theta .
$$

We seek a solution to Eq. (1) in the form [13-15]

$$
\begin{gathered}
\psi(r, \theta)=\psi_{0}(r)+\sum_{n=1}^{\infty} \frac{\varepsilon^{n}}{n !} \psi_{n}(r, \theta), \\
\psi_{n}(r, \theta)=\sum_{k=0}^{n} \psi_{n k}^{(c)}(r) \cos k \theta+\psi_{n k}^{(s)}(r) \sin k \theta .
\end{gathered}
$$

This ansatz is valid for any $r, \theta$, and $\varepsilon$ as long as both series converge, so $\psi_{n}(r, \theta) \sim 1$ is not formally required. Replacing Eqs. (3) in Eq. (1) and collecting terms with equal powers of $\varepsilon$, one finds an equation for $\psi_{0}$,

$$
r^{2} \psi_{0}^{\prime \prime}+r \psi_{0}^{\prime}=-r^{2}\left[\dot{p}_{0}(r)+\dot{Y}_{0}(r)\right],
$$

in which $p_{0}(r)=p\left[\psi_{0}(r)\right], Y_{0}(r)=Y\left[\psi_{0}(r)\right]$, and the primes denote radial derivatives. Likewise, a sequence of linear inhomogeneous equations,

$$
r^{2} \psi_{n k}^{\prime \prime}+r \psi_{n k}^{\prime}+\left[r^{2} \sigma(r)-k^{2}\right] \psi_{n k}=b_{n k},
$$

is found for all other harmonics $\psi_{n k}$ (any of $\psi_{n k}^{(c)}$ or $\psi_{n k}^{(s)}$ ), with $\sigma(r)=\ddot{p}_{0}(r)+\ddot{Y}_{0}(r)$. Each $b_{n k}(r)$ is the $k^{\text {th }}$ harmonic [either $b_{n k}^{(c)}(r)$ or $b_{n k}^{(s)}(r)$ ] of the $n^{\text {th }}$ source term

$$
\begin{aligned}
& \frac{b_{n}(r, \theta)}{r^{2}}=\Delta_{n}^{*} \psi_{0}-\dot{p}_{0} R_{n}-\dot{Y}_{0} \widetilde{R}_{n} \\
& \quad-\sum_{m=1}^{n-1}\left(\begin{array}{c}
n \\
m
\end{array}\right)\left(\dot{p}_{m} R_{n-m}+\dot{Y}_{m} \widetilde{R}_{n-m}-\Delta_{m}^{*} \psi_{n-m}\right),
\end{aligned}
$$


where the $m$-th order differential operator is defined as

$$
\Delta_{m}^{*} \equiv-\frac{1}{r} \frac{\partial}{\partial r}\left(r \widetilde{R}_{m} \frac{\partial}{\partial r}\right)-\frac{1}{r^{2}} \frac{\partial}{\partial \theta}\left(\widetilde{R}_{m} \frac{\partial}{\partial \theta}\right),
$$

while $\dot{p}_{m}=(d / d \varepsilon)^{m} \dot{p}(\psi), \dot{Y}_{m}=(d / d \varepsilon)^{m} \dot{Y}(\psi), R_{m}=$ $(\partial / \partial \varepsilon)^{m} R$, and $\widetilde{R}_{m}=(\partial / \partial \varepsilon)^{m} R^{-1}$, evaluated at $\varepsilon=0$, are radial functions $[14,15]$. Because $\psi(r, \theta)$ is intended near the magnetic axis, the latter is made to coincide with the origin demanding that $\nabla \psi$ vanishes at $r=0$, whence the conditions $\psi_{0}^{\prime}(0)=\psi_{n k}(0)=\psi_{n k}^{\prime}(0)=0$. Letting $\psi_{0}(0)=0$ makes the poloidal flux vanish at the origin also, being thus defined up to an additive constant.

To obtain $\psi_{0}(r)$, the source term in Eq. (4) is taken from the poloidal-plane projection of the force balance, which yields the toroidal current density

$$
-J_{(\phi)}(R, \psi)=R \dot{p}(\psi)+R^{-1} \dot{Y}(\psi)
$$

normalized to $\psi_{\mathrm{N}} /\left(\mu_{0} a^{2} R_{0}\right)$. Its zeroth-order term near the origin can be represented by the series

$$
-\left[\dot{p}_{0}(r)+\dot{Y}_{0}(r)\right]=J_{0}+\frac{J_{2}}{2} r^{2}+\cdots,
$$

where $J_{0}$ is the toroidal current density flowing on axis, $J_{1}=-\left.(d / d r)\left(\dot{p}_{0}+\dot{Y}_{0}\right)\right|_{0}$ vanishes since $d / d r=$ $\psi_{0}^{\prime}(r) d / d \psi$, and $J_{2}=-\left.\left(d^{2} / d r^{2}\right)\left(\dot{p}_{0}+\dot{Y}_{0}\right)\right|_{0}$, whence

$$
\psi_{0}(r)=\frac{J_{0}}{4} r^{2}+\frac{J_{2}}{32} r^{4}+\cdots .
$$

Looking for the next solution $\psi_{1}(r, \theta)$, the source term

$$
b_{1}(r, \theta)=-r^{3}\left[\dot{Y}_{0}-\dot{p}_{0}+\frac{1}{r^{2}} \frac{d}{d r}\left(r^{2} \psi_{0}^{\prime}\right)\right] \cos \theta
$$

taken from (6) shows that $b_{10}^{(c)}$ and $b_{11}^{(s)}$ vanish, whereas

$$
b_{11}^{(c)}=\frac{1}{2}\left[J_{0}-4 \dot{p}_{0}(0)\right] r^{3}+\cdots .
$$

Therefore, from Eq. (5), one finds $\psi_{10}^{(c)}=\psi_{11}^{(s)}=0$ and

$$
\psi_{11}^{(c)}=\frac{1}{16}\left[J_{0}-4 \dot{p}_{0}(0)\right] r^{3}+\cdots .
$$

Similarly, $b_{21}^{(c)}, b_{21}^{(s)}$, and $b_{22}^{(s)}$ are seen to vanish also, while

$$
b_{20}^{(c)}=B_{20} r^{4}+\cdots \quad \text { and } \quad b_{22}^{(c)}=B_{22} r^{4}+\cdots .
$$

As we shall see, the particular values of $B_{20}$ and $B_{22}$ are not important in what follows. Again, Eq. (5) yields

$$
\begin{aligned}
& \psi_{20}^{(c)}(r)=\frac{B_{20}}{16} r^{4}+\cdots, \\
& \psi_{22}^{(c)}(r)=\frac{C_{0}}{2} r^{2}+\frac{2 B_{22}-\sigma(0) C_{0}}{24} r^{4}+\cdots, \\
& \psi_{22}^{(s)}(r)=\frac{S_{0}}{2} r^{2}-\frac{\sigma(0) S_{0}}{24} r^{4}+\cdots,
\end{aligned}
$$

and $\psi_{21}^{(c)}(r)=\psi_{21}^{(s)}(r)=0$, where $C_{0}$ and $S_{0}$ are integration constants whose meaning is discussed below.

Thus far, the solutions in Eqs. (10), (13), and (15), plus their combination in Eqs. (3), are valid for any $r$ and $\varepsilon$, if all series converge and sufficient terms are kept. The procedure outlined previously can thereby proceed to arbitrary powers in $\varepsilon$. However, to get analytically tractable expressions, one must truncate the power series in Eqs. (3) somewhere. This is done dropping all terms $\varepsilon^{\mu} r^{\nu}$ (where $\mu, \nu \geqslant 0$ are integers) smaller than those leading in the combination $\varepsilon^{2} \psi_{2}(r, \theta)$ : First, let $\varepsilon \ll 1$ and $r \ll 1$; Then we find $\varepsilon^{\mu+3} r^{\nu+2} \ll \varepsilon^{2} r^{2}$ and all $\mathcal{O}\left(\varepsilon^{3}\right)$ terms in Eqs. (3), which have the form $\varepsilon^{\mu+3} r^{\nu+2}$, may be dropped when compared to $\varepsilon^{2} r^{2}\left(C_{0} \cos 2 \theta+S_{0} \sin 2 \theta\right) / 4$; Further, the terms $\mathcal{O}\left(r^{4}\right)$ in Eq. (15) are also discarded since $\varepsilon^{2} r^{3+\nu} \ll \varepsilon^{2} r^{2}$; If, in addition, one takes $r / \varepsilon \ll 1$, then $\varepsilon r^{3+\nu} \ll \varepsilon^{2} r^{2}$ and the combination $\varepsilon \psi_{1}(r, \theta)$ may be dropped altogether because its nonvanishing terms [listed in Eq. (13)] are all of the form $\varepsilon r^{3+\nu}$; Likewise, $r^{4+\nu} \ll$ $\varepsilon^{2} r^{2}$ enables all terms $\mathcal{O}\left(r^{4}\right)$ in Eq. (10) to be discarded. Finally, the GS solution to lowest order in aspect ratio $(\varepsilon \ll 1)$ near the axis $(r \ll \varepsilon)$, can be written as

$$
\psi(r, \theta) \simeq \frac{J_{0}}{4} r^{2}+\frac{\varepsilon^{2}}{4}\left(C_{0} r^{2} \cos 2 \theta+S_{0} r^{2} \sin 2 \theta\right) .
$$

Notice that $\varepsilon^{2} C_{0}$ and $\varepsilon^{2} S_{0}$ can be of the same size as $J_{0}$.

Boundary conditions and plasma profiles.-To better understand the physical meaning of the constants

$$
C_{0} \equiv \psi_{22}^{(c) \prime \prime}(0) \quad \text { and } \quad S_{0} \equiv \psi_{22}^{(s) \prime \prime}(0)
$$

in Eq. (15), let us write each $\psi_{n k}(r)$ as $[14,16]$

$$
\frac{\psi_{n k}(r)}{g_{k}(r)}=\frac{\psi_{n k}\left(r_{\mathrm{B}}\right)}{g_{k}\left(r_{\mathrm{B}}\right)}-\int_{r}^{r_{\mathrm{B}}} \frac{d u}{u g_{k}^{2}(u)} \int_{0}^{u} \frac{d v}{v} g_{k}(v) b_{n k}(v),
$$

where $g_{k}(r)$ are homogeneous solutions of Eq. (5) and $\psi_{n k}\left(r_{\mathrm{B}}\right)$ are boundary conditions at $r_{\mathrm{B}}$. Define also

$$
\varsigma=\frac{g_{2}^{\prime \prime}(0)}{g_{2}\left(r_{\mathrm{B}}\right)} \quad \text { and } \quad \xi=\int_{0}^{r_{\mathrm{B}}} \frac{d u}{u} \frac{g_{2}^{\prime \prime}(0)}{g_{2}^{2}(u)} \int_{0}^{u} \frac{d v}{v} g_{2}(v) b_{22}^{(c)}(v),
$$

and recall that $b_{22}^{(s)}$ vanishes whereas $b_{22}^{(c)}$ does not. Hence,

$$
C_{0}=\varsigma \psi_{22}^{(c)}\left(r_{\mathrm{B}}\right)-\xi \quad \text { and } \quad S_{0}=\varsigma \psi_{22}^{(s)}\left(r_{\mathrm{B}}\right) .
$$

Next, let $\psi\left(r_{\mathrm{B}}, \theta\right)$ be the angular distribution of the vacuum poloidal-field flux, inferred from external magnetic measurements $[17,18]$ along some radius $r_{\mathrm{B}}$ beyond the plasma edge. Then, $\psi_{22}^{(c)}\left(r_{\mathrm{B}}\right)$ and $\psi_{22}^{(s)}\left(r_{\mathrm{B}}\right)$ are the leading terms of the complex $2^{\text {nd }}$-order Fourier coefficient

$$
\begin{aligned}
C^{\star}+i S^{\star} & \equiv \frac{1}{\pi} \int_{0}^{2 \pi} \psi\left(r_{\mathrm{B}}, \theta\right) e^{i 2 \theta} d \theta \\
& =\frac{\varepsilon^{2}}{2}\left[\psi_{22}^{(c)}\left(r_{\mathrm{B}}\right)+i \psi_{22}^{(s)}\left(r_{\mathrm{B}}\right)\right]+\mathcal{O}\left(\varepsilon^{3}\right)
\end{aligned}
$$


once the ansatz (3) has been recollected. Thus, $C_{0}$ and $S_{0}$ are proportional to $C^{\star}$ and $S^{\star}$, while $\varsigma$ and $\xi$ in Eq. (20) depend only on the plasma profiles $p(\psi)$ and $Y(\psi)$.

On-axis up-down asymmetry.-Setting $x=r \cos \theta$ and $y=r \sin \theta$, the equilibrium in Eq. (16) becomes

$$
\psi(x, y)=\frac{J_{0}+\varepsilon^{2} C_{0}}{4} x^{2}+\frac{\varepsilon^{2} S_{0}}{2} x y+\frac{J_{0}-\varepsilon^{2} C_{0}}{4} y^{2} .
$$

Its contours are ellipses, all with elongation $\kappa$ (the major to minor-axis ratio) and tilted by some angle $\varphi$ such that

$$
\left|\frac{\kappa^{2}-1}{\kappa^{2}+1}\right|=\left|\frac{\varepsilon^{2}}{J_{0}}\right| \sqrt{C_{0}^{2}+S_{0}^{2}} \quad \text { and } \tan 2 \varphi=\frac{S_{0}}{C_{0}} .
$$

Let a closed curve be defined as $F(x, y)=0$, for some function $F(x, y)$, along which $\nabla F$ does not vanish and $\partial F / \partial y=0$ at two of its points only. These define the limits $x_{1}$ and $x_{2}$ of the curve's projection on the line $y=0$ and such curve can thus be split into a top and bottom branch, respectively $y_{\mathrm{t}}(x)$ and $y_{\mathrm{b}}(x)$, with $y_{\mathrm{b}}(x)<y_{\mathrm{t}}(x)$ for $x_{1}<x<x_{2}$. So, we define the curve's asymmetry as

$$
\eta=\int_{x_{1}}^{x_{2}} \frac{1}{2}\left|y_{\mathrm{b}}(x)+y_{\mathrm{t}}(x)\right| d x / \int_{x_{1}}^{x_{2}}\left[y_{\mathrm{t}}(x)-y_{\mathrm{b}}(x)\right] d x .
$$

Once the top and bottom branches are sorted out from the quadratic form (22), Eq. (24) yields the single value

$$
\eta_{0}=\frac{\varepsilon^{2}\left|S_{0}\right|}{\pi \sqrt{J_{0}^{2}-\varepsilon^{4}\left(C_{0}^{2}+S_{0}^{2}\right)}}
$$

for all ellipses sufficiently near the axis. Additionally, if $\varepsilon^{4}\left(C_{0}^{2}+S_{0}^{2}\right) \ll J_{0}^{2}$, the exact Eq. (25) simplifies to

$$
\eta_{0} \approx \frac{\varepsilon^{2}}{\pi}\left|\frac{S_{0}}{J_{0}}\right|=\frac{2}{\pi}\left|\frac{\varsigma S^{\star}}{J_{0}}\right| .
$$

Moreover, the relations in Eq. (23) suffice to write $\eta_{0}$ as

$$
\eta_{0}=\frac{\eta_{0}^{\max }}{\sqrt{1+\cot ^{2} 2 \varphi}}, \quad \text { with } \quad \eta_{0}^{\max }=\left|\frac{\kappa^{2}-1}{4 \pi \kappa}\right|
$$

the maximum up-down asymmetry at constant $\kappa$, which is attained when $\varphi=\pi / 4$ and therefore $C_{0} \ll S_{0}$.

Since $J_{0}$ is a property of the core, whereas $C_{0}$ and $S_{0}$ depend on the external field, they may be regarded as independent parameters defining the equilibrium shape: Raising $J_{0}$ in Eq. (23) produces increasingly circular magnetic surfaces $(\kappa \sim 1)$ and thus suppresses locally the shaping imposed externally by $C^{\star}$ and $S^{\star}$ via $C_{0}$ and $S_{0}$ in Eqs. (20) and (21); Conversely, the angle $\varphi$ is set by the external shaping only and does not change with $J_{0}$.

The on-axis safety factor in the cylindrical approximation evaluates to $q_{0}=2 a^{2} \psi_{\mathrm{N}}^{-1} B_{0} / J_{0}$. Therefore,

$$
\eta_{0} \approx \varsigma q_{0}\left|\frac{\overline{S^{\star}}}{\pi a^{2} B_{0}}\right|
$$

relates $\eta_{0}$ with the ratio of the poloidal-flux's odd Fourier harmonic $\overline{S^{\star}}=\psi_{\mathrm{N}} S^{\star}$ (in $\mathrm{Wb}$ ) to the flux of the on-axis toroidal field $B_{0}$ through the poloidal section. Of course, Eqs. (25), (26), and (28) are all estimates of the particular definition (24): higher $\eta$ does not necessarily imply more momentum flux.

Analytic Solovev equilibrium. - The example bellow confirms the validity of the ansatz (3) and the approximations leading to Eq. (16); it also illustrates the concepts embodied in Eqs. (20), (19), and (21). Let us consider the analytic Solovev equilibrium [19, 20]

$$
\begin{aligned}
\psi(R, Z)= & -\frac{1}{2} U R^{2}\left(Z^{2}+\frac{1}{4} R^{2}\right)-\frac{1}{2} V\left(R^{2} \log R+Z^{2}\right) \\
& +a_{0}+a_{1} R^{2}+a_{2} Z+a_{3} Z R^{2} \\
& +a_{4} R^{2}\left(R^{2}-4 Z^{2}\right)+a_{5}\left(Z^{2}-R^{2} \log R\right) \\
& +a_{6}\left[2 Z^{4}-9 Z^{2} R^{2}+3 R^{2}\left(R^{2}-4 Z^{2}\right) \log R\right]
\end{aligned}
$$

which solves Eq. (1) for constant $\dot{p}(\psi)=\varepsilon^{2} U$ and $\dot{Y}(\psi)=$ $\varepsilon^{2} V$, with $-\varepsilon^{-2} \Delta^{*}=\partial_{R} R^{-1} \partial_{R}+R^{-1} \partial_{Z Z}^{2}$. Here, each arbitrary $a_{n}$ multiplies an homogeneous GS solution. Of these coefficients, $a_{0}, a_{1}$, and $a_{2}$ are taken from the conditions $\psi(1,0)=\nabla \psi(1,0)=0$, while $a_{3}=-\frac{1}{4} S_{0}$,

$$
\begin{aligned}
& a_{4}=2^{-10}\left[23\left(V-C_{0}\right)-9 U-27 c_{1}-15 c_{2}\right], \\
& a_{5}=2^{-7}\left[7 V-9\left(U+c_{1}\right)-39 C_{0}-3 c_{2}\right], \quad \text { and } \\
& a_{6}=2^{-8}\left(C_{0}-U-V+c_{1}+c_{2}\right)
\end{aligned}
$$

are written in terms of four new arbitrary constants: $S_{0}$, $C_{0}, c_{1}$, and $c_{2}$. The transformation (2), after setting $\varepsilon^{2} U=\dot{p}_{0}$ and $\varepsilon^{2}(U+V)=-J_{0}$, turns Eq. (29) into

$$
\begin{aligned}
& \psi(r, \theta)=\frac{J_{0}}{4} r^{2}-\varepsilon \frac{J_{0}-4 \dot{p}_{0}}{16} r^{3} \cos \theta+\frac{\varepsilon^{2}}{2}\left[\frac{B_{20}}{16} r^{4}\right. \\
& \left.+\left(\frac{C_{0}}{2} r^{2}+\frac{B_{22}}{12} r^{4}\right) \cos 2 \theta+\frac{S_{0}}{2} r^{2} \sin 2 \theta\right]+\mathcal{O}\left(\varepsilon^{3}\right),
\end{aligned}
$$

where $B_{20}=-\frac{1}{4}\left(J_{0}+8 \dot{p}_{0}\right)$ and $B_{22}=-\frac{3}{8}\left(J_{0}+4 \dot{p}_{0}\right)$. As expected, its harmonics match the leading terms in the general Eqs. (10), (13), and (15), since $\dot{p}_{0}$ and $\dot{Y}_{0}$ are here constant [and thus $\sigma(r)=0$ ]. One finds also $g_{2}(r) \propto r^{2}$, whence $\varsigma=2 r_{\mathrm{B}}^{-2}$ and $\xi=\frac{1}{6} B_{22} r_{\mathrm{B}}^{2}$ from Eqs. (19). The exact solution (31), evaluated at $r_{\mathrm{B}}$, clearly illustrates how $C_{0}$ and $S_{0}$ relate with $C^{\star}$ and $S^{\star}$ via the boundary conditions, $\psi_{22}^{(c)}\left(r_{\mathrm{B}}\right)$ and $\psi_{22}^{(s)}\left(r_{\mathrm{B}}\right)$, and Eqs. (20) and (21).

Numerical tokamak equilibria.- GS solutions are next computed for parameters typical of the Tokamak à Configuration Variable (TCV) [21], where evidence of momentum flux induced by up-down asymmetry has been reported [22, 23]. Convergent equilibria for finite $\varepsilon$ and $r$ are obtained by dropping $\mathcal{O}\left(\varepsilon^{9}\right)$ terms in the ansatz (3), with Eqs. (5) and (4) yielding a set of equations for 

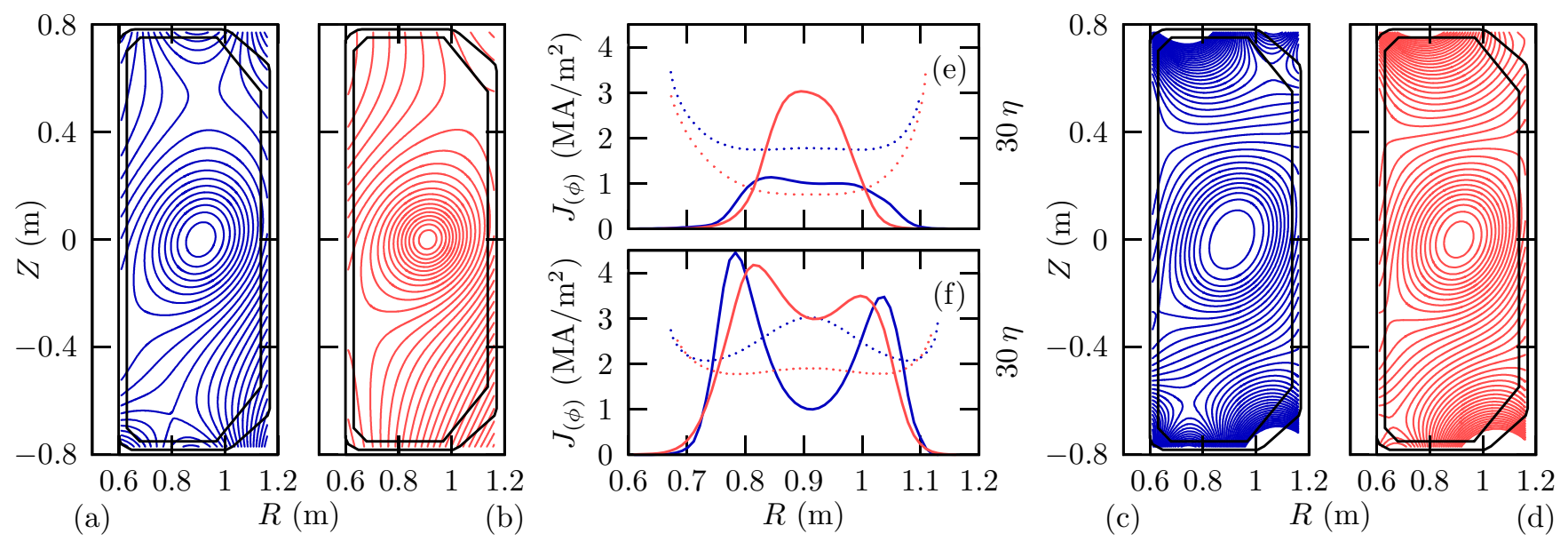

FIG. 1. Magnetic equilibria for small [(a) and (b), left] and large [(c) and (d), right] $I_{\mathrm{p}}$ : Equidistant contours start at the same value and are drawn in dark [(a) and (c)] and light tones [(b) and (d)] for low and high $J_{0}$ respectively; Midplane profiles of $J_{(\phi)}$ (solid lines) and $\eta$ (dotted lines) for small [(e), top] and large [(f), bottom] $I_{\mathrm{p}}$ scenarios follow the same tone rule.

TABLE I. Plasma parameters for numerical TCV-like equilibria: $I_{\mathrm{p}}(\mathrm{kA}), \psi_{\mathrm{N}}(\mathrm{Wb}), P_{0}(\mathrm{kPa})$, and $J_{0}\left(\mathrm{MA} / \mathrm{m}^{2}\right)$; other parameters are dimensionless.

\begin{tabular}{lrlrrrrrrr}
\hline \hline & $I_{\mathrm{p}}$ & $\psi_{\mathrm{N}}$ & $P_{0}$ & $J_{0}$ & \multicolumn{1}{c}{$\alpha$} & $\gamma$ & $\left.\psi_{22}^{(c)}\right|_{\mathrm{B}}$ & $\left.\psi_{22}^{(s)}\right|_{\mathrm{B}}$ & $\left.\psi_{44}^{(c)}\right|_{\mathrm{B}}$ \\
\hline (a) & 76 & 0.01 & 3.74 & 1 & 5.93 & 1.80 & 4 & 5 & 10 \\
(b) & 76 & 0.01 & 3.74 & 3 & -7.25 & 1.40 & 3 & 5 & 10 \\
(c) & 355 & 0.03 & 17.51 & 1 & 32.54 & 2.08 & 6 & 6 & 200 \\
(d) & 355 & 0.03 & 17.51 & 3 & 10.80 & 1.44 & 6 & 6 & 200 \\
\hline \hline
\end{tabular}

$n, k \leqslant 8$ which are solved using the plasma models

$$
\begin{gathered}
p(\psi)=\frac{1}{2} P_{0}\left[1-\tanh \left(P_{1} \psi-P_{2}\right)\right] \text { and } \\
\dot{p}(\psi)+\dot{Y}(\psi)=-\left(J_{0}+\alpha \psi\right) \exp \left[-(\gamma \psi)^{2}\right]
\end{gathered}
$$

where $P_{0}, P_{1}=4.2, P_{2}=2.65, J_{0}, \alpha$, and $\gamma$ are constants.

For a given plasma current $I_{\mathrm{p}}$, the normalized on-axis pressure $P_{0}$ is chosen to keep the normalized $\beta[13]$

$$
\beta_{\mathrm{N}}=a B_{0} \frac{\beta}{I_{\mathrm{p}}} \sim \frac{2 \psi_{\mathrm{N}}^{2}}{a B_{0} R_{0}^{2}} \frac{P_{0}}{I_{\mathrm{p}}}=0.02 \mathrm{Tm} / \mathrm{MA}
$$

fixed, with $a=0.24 \mathrm{~m}, R_{0}=0.91 \mathrm{~m}$, and $B_{0}=1.4 \mathrm{~T}$. The remaining parameters are chosen to fit the plasma inside the vessel $\left(\alpha, \gamma\right.$, and $\left.\psi_{\mathrm{N}}\right)$ and to adjust its shape $\left[\left.\psi_{22}^{(c)}\right|_{\mathrm{B}},\left.\psi_{22}^{(s)}\right|_{\mathrm{B}}\right.$, and $\left.\psi_{44}^{(c)}\right|_{\mathrm{B}}$ at $r_{\mathrm{B}}=1$, all other $\left.\psi_{n k}\right|_{\mathrm{B}}=0$, with $\left.\left.\psi_{n k}\right|_{\mathrm{B}} \equiv \psi_{n k}\left(r_{\mathrm{B}}\right)\right]$. These are listed in Table I for two scenarios (small and large $I_{\mathrm{p}}$ ), each with two configurations (low and high $J_{0}$ ). The corresponding numerical equilibria are plotted in Fig. 1.

The profiles in Figs. 1 (e) and (f) agree with Eq. (25): Similar values of $\eta$ at the edge result in core asymmetry which is lower for higher values of $J_{0}$. This asymmetry suppression may be thought of as a competition between
TABLE II. On-axis asymmetry: estimated values $\left(\eta_{0}\right)$ against numerical ones $[\eta(0)]$. All variables are dimensionless.

\begin{tabular}{lcclll}
\hline \hline & $J_{0}$ & $\varsigma$ & $S^{\star}$ & $\eta_{0}$ & $\eta(0)$ \\
\hline (a) & 6.58 & 3.41 & 0.174 & 0.0574 & 0.0593 \\
(b) & 19.7 & 4.44 & 0.174 & 0.0249 & 0.0252 \\
(c) & 2.07 & 1.50 & 0.209 & 0.0962 & 0.101 \\
(d) & 6.21 & 2.87 & 0.209 & 0.0613 & 0.0636 \\
\hline \hline
\end{tabular}

the imposed external field, which affects $C_{0}$ and $S_{0}$, and the symmetric field induced locally by $J_{0}$. Therefore, to increase the asymmetry on axis, one must decrease $J_{0}$ without making $I_{\mathrm{p}}$ dwindle to undesirably low values. Indeed, for any current-density profile with a global maximum on axis, the constraint $\left|J_{0}\right| \gtrsim\left|I_{\mathrm{p}}\right| /\left(\pi a^{2}\right)$ places a limit on how much $J_{0}$ can be reduced for a given $I_{\mathrm{p}}$. This problem is avoided using hollow current-density profiles, as in Fig. 1 (f), where most of the current flows off axis. Besides affording lower $J_{0}$ values (among other advantages for confinement and stability [24]), hollow current profiles induce an asymmetry build-up towards the core: the value of $\eta$ on axis grows to a local maximum and may become larger than the one at the edge.

The estimate in Eq. (26) is tested in Table II, where the values of $J_{0}, \varsigma$, and $S^{\star}$ [with $\varsigma$ and $S^{\star}$ computed from Eqs. (19) and (21)] for each configuration in Table I are used to evaluate $\eta_{0}$. The latter is found to agree rather well with the numerical value $\eta(0)$, which is computed directly from Eq. (24) for magnetic surfaces near the axis.

Lastly, a large number ( $\sim 270)$ of equilibria are computed for a broader set of parameters, with the on-axis current density ranging between 1 and $3 \mathrm{MA} / \mathrm{m}^{2}$, while $I_{\mathrm{p}}=75,200$, and $350 \mathrm{kA}$. Other parameters are varied within reasonable bounds to keep the plasma inside the 


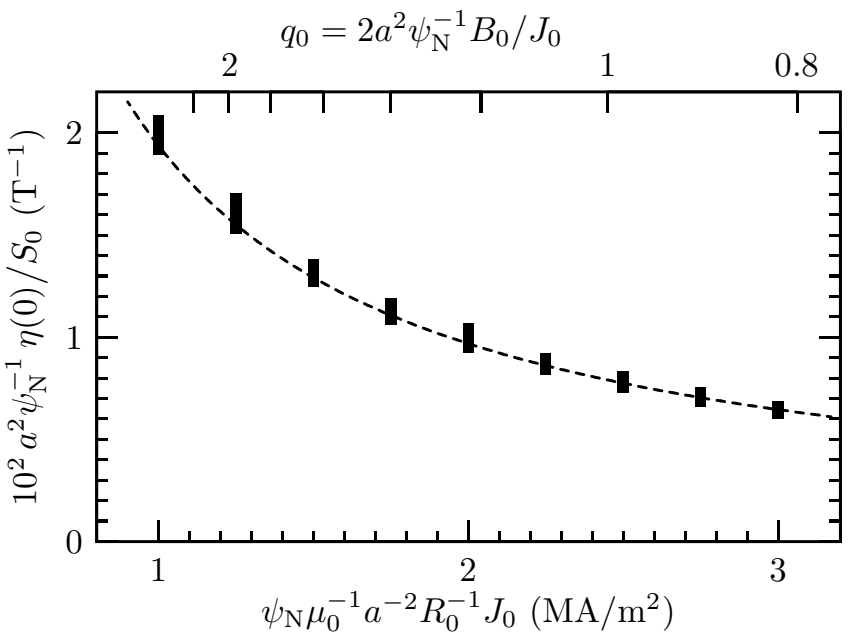

FIG. 2. Ratio of computed $\eta(0)$ to $S_{0}$ (squares) and analytic curve $\eta(0) / S_{0} \approx \varepsilon^{2} /\left(\pi J_{0}\right)$ (dashed line).

vessel. For each equilibrium, $S_{0}$ is evaluated as $\psi_{22}^{(s) \prime \prime}(0)$ and the ratio $\eta(0) / S_{0}$ is plotted against $J_{0}$ in Fig. 2 . The agreement with Eq. (26) is manifest. Also, the dispersion of computed results around the curve, due to nonzero $\varepsilon^{4}\left(C_{0}^{2}+S_{0}^{2}\right)$ in Eq. (25), is higher for smaller $J_{0}$.

Conclusions.-An analytic up-down asymmetric equilibrium was developed near the magnetic axis, which depends on three parameters only: $J_{0}, C_{0}$, and $S_{0}$. The asymmetry was quantified and found to be established by the ratio of $S_{0} \propto \varsigma S^{\star}$ (which measures the ability of the external-field's odd perturbation $S^{\star}$ to propagate into the core) to the on-axis current density $J_{0}$. The results were tested with a Solovev equilibrium and with numerical equilibria computed for TCV parameters. An important finding is that hollow current profiles (and thus reverse magnetic shear) are seen to improve on-axis asymmetry values, which can even be larger than those at the edge. These findings may enhance the intrinsic rotation in the core, thus improving plasma confinement.

This work was supported by EURATOM and carried out within the framework of the European Fusion Development Agreement. IST activities were also supported by "Fundação para a Ciência e Tecnologia" through project Pest-OE/SADG/LA0010/2011. The views and opinions expressed herein do not necessarily reflect those of the European Commission. J. Ball and F. I. Parra were supported by US DOE Grant No. DE-SC008435.

* par@ipfn.ist.utl.pt

[1] E. Doyle, W. Houlberg, Y. Kamada, V. Mukhovatov, T. Osborne, A. Polevoi, G. Bateman, J. Connor, J. Cordey, T. Fujita, X. Garbet, T. Hahm, L. Horton, A. Hubbard, F. Imbeaux, F. Jenko, J. Kinsey, Y. Kishi- moto, J. Li, T. Luce, Y. Martin, M. Ossipenko, V. Parail, A. Peeters, T. Rhodes, J. Rice, C. Roach, V. Rozhansky, F. Ryter, G. Saibene, R. Sartori, A. Sips, J. Snipes, M. Sugihara, E. Synakowski, H. Takenaga, T. Takizuka, K. Thomsen, M. Wade, H. Wilson, I. T. P. T. Group, I. C. Database, M. T. Group, I. Pedestal, and E. T. Group, Nucl. Fusion 47, S18 (2007).

[2] K. H. Burrell, Phys. Plasmas 4, 1499 (1997).

[3] H. Biglari, P. H. Diamond, and P. W. Terry, Phys. Fluids B 2, 1 (1990).

[4] R. E. Waltz, G. D. Kerbel, J. Milovich, and G. W. Hammett, Phys. Plasmas 2, 2408 (1995).

[5] E. G. Highcock, M. Barnes, A. A. Schekochihin, F. I. Parra, C. M. Roach, and S. C. Cowley, Phys. Rev. Lett. 105, 215003 (2010).

[6] M. Barnes, F. I. Parra, E. G. Highcock, A. A. Schekochihin, S. C. Cowley, and C. M. Roach, Phys. Rev. Lett. 106, 175004 (2011).

[7] A. G. Peeters and C. Angioni, Phys. Plasmas 12, 072515 (2005).

[8] F. I. Parra, M. Barnes, and A. G. Peeters, Phys. Plasmas 18, 062501 (2011).

[9] A. Peeters, C. Angioni, A. Bortolon, Y. Camenen, F. Casson, B. Duval, L. Fiederspiel, W. Hornsby, Y. Idomura, T. Hein, N. Kluy, P. Mantica, F. Parra, A. Snodin, G. Szepesi, D. Strintzi, T. Tala, G. Tardini, P. de Vries, and J. Weiland, Nucl. Fusion 51, 094027 (2011).

[10] Y. Camenen, A. G. Peeters, C. Angioni, F. J. Casson, W. A. Hornsby, A. P. Snodin, and D. Strintzi, Phys. Rev. Lett. 102, 125001 (2009).

[11] Y. Camenen, A. G. Peeters, C. Angioni, F. J. Casson, W. A. Hornsby, A. P. Snodin, and D. Strintzi, Phys. Plasmas 16, 062501 (2009).

[12] J. P. Freidberg, Ideal Magnetohydrodynamics (Plenum, 1987).

[13] J. Wesson, Tokamaks, 2nd ed. (Oxford University Press, 1997).

[14] P. Rodrigues and J. P. S. Bizarro, Phys. Plasmas 11, 186 (2004).

[15] P. Rodrigues and J. P. S. Bizarro, Phys. Plasmas 16, 022505 (2009).

[16] P. Rodrigues and J. P. S. Bizarro, Phys. Rev. Lett. 95, 015001 (2005).

[17] D. K. Lee and Y. K. M. Peng, J. Plasma Phys. 25, 161 (1981).

[18] F. Sartori, A. Cenedese, and F. Milani, Fusion Eng. Des. 66-68, 735 (2003).

[19] L. S. Solovev, Sov. Phys. JETP 26, 400 (1968).

[20] A. J. Cerfon and J. P. Freidberg, Phys. Plasmas 17, $032502(2010)$.

[21] F. Hofmann, J. B. Lister, W. Anton, S. Barry, R. Behn, S. Bernel, G. Besson, F. Buhlmann, R. Chavan, M. Corboz, M. J. Dutch, B. P. Duval, D. Fasel, A. Favre, S. Franke, A. Heym, A. Hirt, C. Hollenstein, P. Isoz, B. Joye, X. Llobet, J. C. Magnin, B. Marletaz, P. Marmillod, Y. Martin, J. M. Mayor, J. M. Moret, C. Nieswand, P. J. Paris, A. Perez, Z. A. Pietrzyk, R. A. Pitts, A. Pochelon, R. Rage, O. Sauter, G. Tonetti, M. Q. Tran, F. Troyon, D. J. Ward, and H. Weisen, Plasma Phys. Control. Fusion 36, B277 (1994).

[22] Y. Camenen, A. Bortolon, B. P. Duval, L. Federspiel, A. G. Peeters, F. J. Casson, W. A. Hornsby, A. N. Karpushov, F. Piras, O. Sauter, A. P. Snodin, and G. Szepesi, Phys. Rev. Lett. 105, 135003 (2010). 
[23] Y. Camenen, A. Bortolon, B. P. Duval, L. Federspiel, A. G. Peeters, F. J. Casson, W. A. Hornsby, A. N. Karpushov, F. Piras, O. Sauter, A. P. Snodin, G. Szepesi, and the TCV Team, Plasma Phys. Control. Fusion 52,
124037 (2010).

[24] C. Kessel, J. Manickam, G. Rewoldt, and W. M. Tang, Phys. Rev. Lett. 72, 1212 (1994). 